\title{
The effect of continuous, nonlinearly transformed visual feedback on rapid aiming movements
}

\author{
Martina Rieger · Willem B. Verwey • \\ Cristina Massen
}

Received: 28 November 2007 / Accepted: 2 July 2008/Published online: 13 August 2008

(C) The Author(s) 2008. This article is published with open access at Springerlink.com

\begin{abstract}
We investigated the ability to adjust to nonlinear transformations that allow people to control external systems like machines and tools. Earlier research (Verwey and Heuer 2007) showed that in the presence of just terminal feedback participants develop an internal model of such transformations that operates at a relatively early processing level (before or at amplitude specification). In this study, we investigated the level of operation of the internal model after practicing with continuous visual feedback. Participants executed rapid aiming movements, for which a nonlinear relationship existed between the target amplitude seen on the computer screen and the required movement amplitude of the hand on a digitizing tablet. Participants adjusted to the external transformation by developing an internal model. Despite continuous feedback, explicit awareness of the transformation did not develop and the internal model still operated at the same early processing level as with terminal feedback. Thus with rapid aiming movements, the type of feedback may not matter for the locus of operation of the internal model.
\end{abstract}

M. Rieger $(\bowtie) \cdot$ C. Massen

Department of Psychology,

Max Planck Institute for Human Cognitive and Brain Sciences, Stephanstrasse 1a, 04103 Leipzig, Germany

e-mail: rieger@cbs.mpg.de

C. Massen

e-mail: massen@cbs.mpg.de

W. B. Verwey

Cognitive Psychology and Ergonomics, University of Twente,

P.O. Box 217, 7500 AE Enschede, The Netherlands

e-mail: W.B.Verwey@gw.utwente.nl
Keywords Psychomotor performance .

Visual motor coordination - Tool use behavior .

Psychological adaptation Perceptual motor performance

\section{Introduction}

Many everyday skills involving the control of machines or the use of tools require people to adjust their movements to an external transformation between their own movements and the resulting consequences in external space. People are able to adapt quite well to such transformations (Imamizu et al. 2000; Wolpert et al. 1995; Ghahramani et al. 1996; Kagerer et al. 1997), although some transformations are easier to learn than others are. In general, transformations scaling gain are easy to adapt to (Bedford 1994; Bock and Burghoff 1997; Seidler et al. 2001; Rieger et al. 2005). However, transformations involving nonlinear relationships are more difficult to acquire (Heuer and Hegele 2007; Verwey and Heuer 2007). The present research investigates the human ability to adjust to such nonlinear transformations.

An everyday example of adjustment to an external nonlinear transformation is steering a car. Here, an internal model of the transformation allows determining the steering wheel rotation required to attain a goal specified in terms of lane position and heading angle. The ability to develop internal models of transformations implies that people are able to control systems directly in terms of system output, even before they start moving (i.e., in open loop mode, which is fast), rather than that they need to continuously attend to the system output and correct deviations from the intended output (closed loop, which is slow; e.g., Heuer and Hegele 2007; Massen and Prinz 2007). Developing internal models of nonlinear transformations represents an important aspect of perceptual-motor integration, which is one of the key 
motor control processes (e.g., Willingham 1998). Theoretically, one can distinguish several levels at which acquired internal models of nonlinear transformations might operate. Following Verwey and Heuer (2007), we distinguish between amplitude specification and trajectory generation, processes that are accompanied by other perceptual, cognitive and execution processes (see Fig. 1). We assume that perceptual processes identify the target amplitude. Then amplitude specification translates the perceived target amplitude into a code representing the amplitude that a limb will have to move. The amplitude code is then translated into a movement with a specific velocity-time profile. Finally, movement execution processes take place (for a detailed description of the model see Verwey and Heuer 2007).

Given this serial processing model, the question arises at what level acquired internal models operate. To investigate the locus of adjustment to nonlinear transformations between movement space and external space under conditions of terminal feedback, Verwey and Heuer (2007) had participants move a mouse-like device in order to control a cursor on a screen to a target position. Without telling the participants, the relationship between cursor movement amplitude and mouse movement amplitude was exponential in Experiment 1 and logarithmic in Experiment 2 (while it was linear for the control groups). The results of both experiments showed that participants adjusted well to the external transformation, indicating that they had developed an internal model. Furthermore, results indicated that the internal model operates at an early processing level that either precedes amplitude specification or has become part of it.

The present study investigates adjustment to a nonlinear transformation under conditions of continuous feedback: the cursor is continuously presented during motion. The reason to study this is that continuous cursor presentation may reduce advance programming and make participants rely more on online corrections, thus inducing adjustment at a later processing level than amplitude specification (Heuer 2003). In fact, several studies suggest that the mechanisms of adaptation may be different depending on the type of feedback. For example, intermanual transfer of aftereffects in prism adaptation occurred only when participants were provided with terminal feedback but not with continuous feedback (Cohen 1967). This could indicate that the locus of the adjustment to the prism distortion occurred at an earlier level with terminal feedback than with continuous feedback. Similarly, after effects of adaptation to a directional bias between the locations of the cursor and the hand appeared more robust when feedback in the adaptation phase was terminal instead of continuous (Bernier et al. 2005). Detailed analyses suggested that while terminal feedback induced offline recalibration of proprioceptive and visual feedback (implying adjustment of amplitude specification), continuous feedback made participants adjust by online corrections (implying adjustment at the motor execution level). Furthermore, several studies showed that when continuous feedback involves a curved distortion of the path of the cursor, participants compensate by moving their hand along a trajectory with the opposite distortion, thus resulting in a straight cursor trajectory again (Flanagan and Rao 1995; Wolpert et al. 1995). Here the internal model seems to operate at the level of trajectory generation or movement execution. Hence, with continuous feedback the operation of the internal model may occur at a processing level that is later (i.e., at trajectory generation or movement execution) than with terminal feedback. It is, however, unknown whether continuous feedback leads to a late operation of the internal model in all situations. Specifically, rapid movements may not allow for late operation of the internal model (i.e., at the execution level), because there is no enough time for online adjustment.

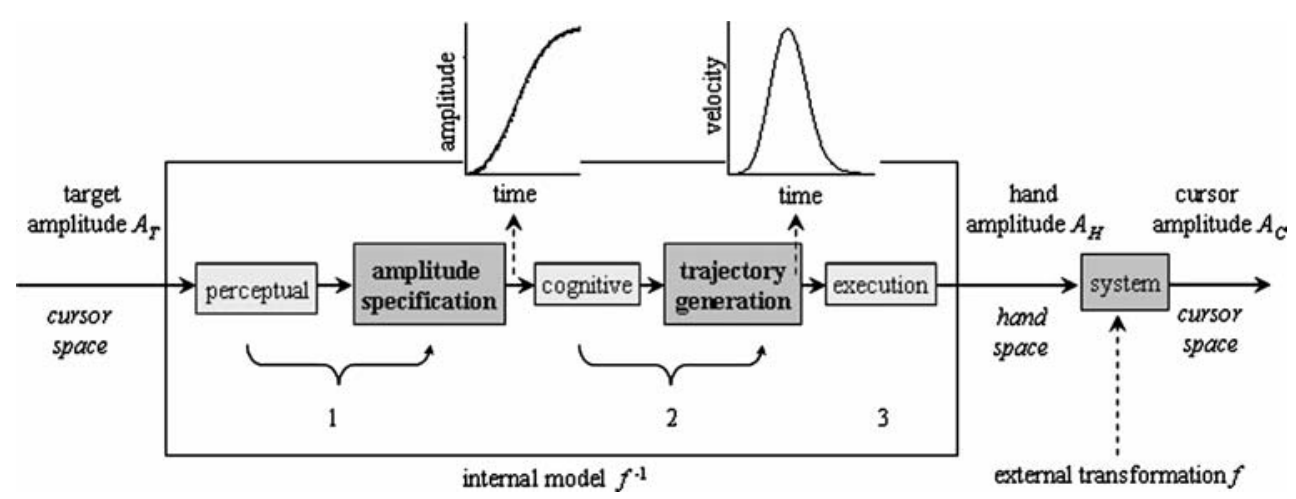

Fig. 1 In a serial information processing model, amplitude specification and trajectory generation are assumed to involve separable processes in human motor control. The internal model of the external transformation, responsible for the transition from cursor space to hand space, may operate before amplitude specification, or be part of amplitude specification (Level 1), operate at processes in between amplitude specification and trajectory generation (Level 2), be part of trajectory generation, or it may exert its effect on processes following trajectory generation (Level 3) 
In the present study, participants rapidly moved a pen from left to right across the surface of a digitizing tablet. Pen movements were subjected to either a nonlinear transformation (cursor-same and hand-same groups) or not (no-transformation group). Participants first performed the task with continuous feedback. This was done to test whether the internal model of the nonlinear transformation operates at a 'late' level under continuous feedback in contrast to the previous study where it operated at an 'early' level with terminal feedback (Verwey and Heuer 2007). Subsequently, participants performed the task with terminal feedback. If the operation of the internal model is at a late processing level under continuous feedback, it may still change to an earlier processing level when the type of feedback is changed to terminal.

\section{Methods}

\section{Participants}

Sixty right-handers participated, of which 16 were excluded for various reasons (see below). Thirteen remained in the no-transformation group (mean age 23.5, $\mathrm{SD}=4.5 ; 12$ females), 15 in the cursor-same group (age 24.0, $\mathrm{SD}=4.2$; 13 females), and 16 in the hand-same group (age 24.0, $\mathrm{SD}=$ $4.3 ; 13$ females). They were paid $€ 21.00$ for participation. The local ethics committee approved the study and all participants gave informed consent.

\section{Apparatus and stimuli}

Participants sat at a table in a dimly lit room with their heads supported by a chin rest. Movements were recorded using a Wacom Ultrapad A3 digitizing tablet at a resolution of 500 pixels per $\mathrm{cm}$ and at a rate of $100 \mathrm{~Hz}$. A cover hid the hand from view. The participants viewed a $17 \mathrm{in.}$ VGA monitor at a distance of $50 \mathrm{~cm}$. The screen showed a black vertical line across its entire height at the left side (i.e., the start line), and a gray vertical line $130 \mathrm{~mm}$ (no-transformation and cursor-same groups) or $208.3 \mathrm{~mm}$ (hand-same group) to the right of the start line, indicating the default target line. The cursor was a small red dot (diameter: 4 $\mathrm{mm}$ ) presented on a white background. Trials were started by positioning the pen at the start location, corresponding to a visually presented square in the middle of the start line.

The target line was a second vertical black line that was presented at different times preceding movement onset. The default target line remained visible when the target line was displayed. Participants were instructed to prepare a movement to the default target line and to modify movement preparation if the black target line appeared at another position. In the first phase of the experiment, the cursor remained visible during motion (continuous feedback). In the subsequent phase, it disappeared as soon as movement began and re-appeared when it had ended (terminal feedback).

Participants of the no-transformation control group performed the task without an external transformation. That is, the amplitude of cursor motion $\left(A_{\mathrm{C}}\right)$ was the same as the amplitude of hand (or pen) movement $\left(A_{\mathrm{H}}\right.$ ), i.e., $A_{\mathrm{C}}=A_{\mathrm{H}}$. Consequently, visuomotor gain was 1.0 for all movement amplitudes. Participants of the two experimental groups had an exponential pen-cursor transformation. We used:

$A_{\mathrm{C}}=f\left(A_{\mathrm{H}}\right)=a+b e^{c A_{\mathrm{H}}}$

with $a=233, b=-381.8$, and $c=-0.0211$. The parameters were chosen such that two target amplitudes (30 and $230 \mathrm{~mm}$ ) were identical in cursor and hand space (visuomotor gain of 1.0 as in the no-transformation condition, see the dashed lines in the rightmost frames of Fig. 3). Because in Eq. $1 A_{\mathrm{C}}$ does not equal zero when $A_{\mathrm{H}}$ does, Eq. 1 was used only for $A_{\mathrm{C}} \geq 0 \mathrm{~mm}$ and replaced by $A_{\mathrm{C}}=0$ for cursor amplitudes below $0 \mathrm{~mm}$. This appeared not to be noticed by the participants.

The task included five target amplitudes. In the cursorsame group, participants saw the same target amplitudes on the screen as participants in the no-transformation group, that is, amplitudes of $30,80,130,180$, and $230 \mathrm{~mm}$. These participants were to move their hand for 30, 43.4, 62.2, 93.7, and $230 \mathrm{~mm}$, respectively, to reach these targets. Participants in the hand-same group were to produce the same five hand-movement amplitudes as the no-transformation participants $(30,80,130,180,230 \mathrm{~mm})$. They were shown target amplitudes of $30,162.3,208.3,224.4$, and $230 \mathrm{~mm}$ in cursor space. We included both a cursor-same and a handsame group in the experiment, because some statistical comparisons are not possible with each of the transformation groups. The intermediate amplitudes of the hand-same group are different from the other groups in cursor space, whereas the intermediate amplitudes of the cursor-same group are different from the two other groups in hand-space.

Participants were to initiate their movements in synchrony with onset of the last of four predictable $20 \mathrm{~ms}$ tones increasing in pitch $(1,000,1,333,1,666,2,000 \mathrm{~Hz})$, separated by $500 \mathrm{~ms}$ (see Fig. 2; timed response method, Hening et al. 1988). They then moved the pen rightward with their right hand across the digitizing tablet. The target line appeared at one of six times preceding, and in one case, following the onset of the fourth tone with a stimulus onset interval (SOA) of $920,670,420,295,170,45$, or -80 $\mathrm{ms}$. If the movement started more than $80 \mathrm{~ms}$ before or after onset of the fourth tone, participants were informed of a timing error (though movements with onsets within a $\pm 160 \mathrm{~ms}$ window were analyzed). Slow movements were movements with an average velocity below $80 \%$ of the 


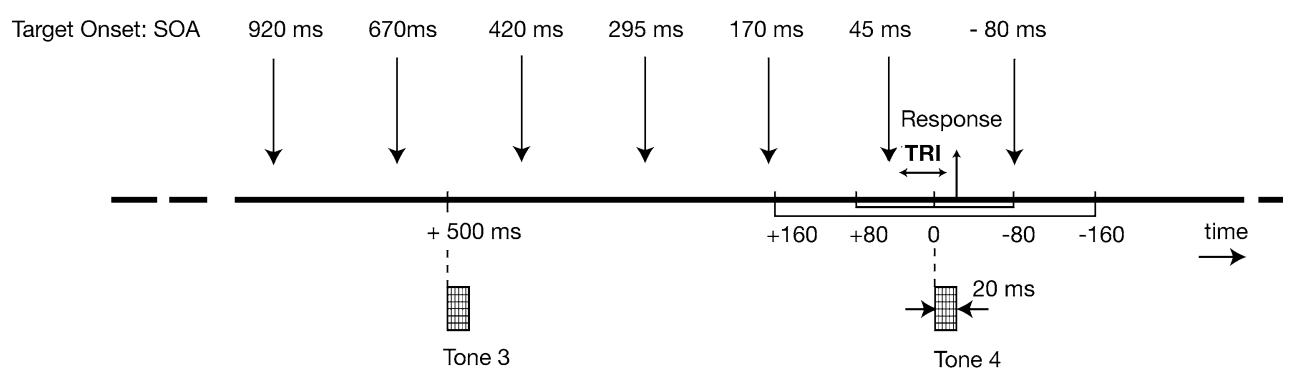

Fig. 2 Temporal relationship between the countdown tones, the various stimulus onset asynchronies (SOAs) relative to onset of Tone 4 , and the movement initiation windows $(< \pm 80$ and $\pm 160 \mathrm{~ms})$. Shown is a trial with a fictive movement ('Response') onset time 25

mean velocity for a certain amplitude length obtained in a pilot study. Irregular movements had more than one velocity peak. Slow and irregular movements also produced an error message.

After each feedback condition, participants were presented with a sheet of paper on which the five target amplitudes they had seen in cursor space were presented as lines with the same length as seen earlier on the screen. They were requested to draw a line below each target amplitude representing the amplitude they thought they had moved their hand.

\section{Procedure}

Each participant obtained a written task instruction that stressed the importance of starting to move at the onset of the fourth tone and to move smoothly (i.e., to avoid corrective submovements). Participants were instructed to bring the cursor to the target position while starting movement at the indicated time. They were informed that reaching the target position was not possible when the target line changed position only briefly before movement onset. In order to obtain well-defined endpoints of the movements, participants were instructed to remain on the final position for a short time. In addition, they were told that the relationship between hand and cursor amplitude would vary with pen position.

Participants performed 30 blocks of 35 trials in which the cursor remained visible during motion. The last 15 blocks (525 trials) were analyzed. Their subjective knowledge of the transformation was then tested with the paper-and-pen-test. Next, they performed in 10 blocks (350 trials) without the cursor being visible during motion and were again tested with the paper-and-pen test.

\section{Data analyses}

The vertical (Y-) position of the pen on the tablet was neglected and data analysis was restricted to the $\mathrm{X}$-coordinates. First, the position data were interpolated to yield a ms after onset of Tone 4 , in response to onset of the target line $45 \mathrm{~ms}$ before onset of Tone 4 (i.e., SOA $45 \mathrm{~ms}$ ). This yields a positive targetresponse interval (TRI) of $45+25=70 \mathrm{~ms}$

constant sample rate of $100 \mathrm{~Hz}$ because there was some variation in the sampling period of the digitizing tablet (7-13 ms). Then the kinematic data were smoothed using a nonlinear algorithm (Mottet et al. 1994). Movement initiation was defined as the first of four consecutive samples (30 ms), in which participants moved more than $0.5 \mathrm{~mm}$ on the digitizing tablet. Movement completion was defined as the participant moving less than $0.5 \mathrm{~mm}$ for 16 consecutive samples (i.e., $150 \mathrm{~ms}$ ).

\section{Results}

Sixteen of the 60 participants were excluded from analysis: 6 of them had more than $40 \%$ invalid trials, 4 responded with similar movements even with sufficient preparation time, 3 did not have sufficient trials in all bins, and with 3 there were technical problems. Individual trials were excluded from analysis when participants started more than $160 \mathrm{~ms}$ before or after onset of the fourth tone. Furthermore, trials with either outlying movement times ( \pm 3 SDs for a specific amplitude), or more than one velocity peak were excluded. On average there were $81.3 \%(\mathrm{SD}=8.3 \%)$ valid trials per participant.

To reduce the effect of variations in movement onset times, movements were classified according to the actual interval between target onset and movement onset (targetresponse interval or TRI, see Fig. 2). TRI bins were chosen such that there was a reasonable number of bins in the important 0-400 ms range, and that each of them included a fair number of observations. This resulted in seven bins with the same boundaries in the continuous and terminal feedback condition (bin [range] in ms: -105 [-240, ..,29], 75 [30,..,119], 165 [120,..,209], 255 [210,..,299], 400 [300,..,499], 625 [500,..,749], 925 [750,..,1,100]). Each of these 7 bins contained between 9 and $19 \%$ of the data in that amplitude condition.

Below, we will focus on the results from the continuous feedback condition and then report the results of the transfer test with terminal feedback. 
The internal model

Movement accuracy was better with increasing TRI and reached asymptote at about $400 \mathrm{~ms}$ (cf. Hening et al. 1988). The amplitude data of bins 400, 625, and $925 \mathrm{~ms}$ were, therefore, averaged to determine adjustments to the external nonlinearity that had not been influenced by insufficient preparation time. Figure 3 (left and middle frames) shows actual movement amplitudes in cursor and in hand space with continuous feedback (upper frames). The right frames of Fig. 3 depict the acquired internal model of the transformation from cursor to hand space, which approximated the inverse function of the external transformation (dashed lines in right frames).

Only movement amplitudes produced at the two target amplitudes of 30 and $230 \mathrm{~mm}$ are directly comparable across groups because the visuomotor gain at these amplitudes was 1.0 for all three groups of participants. Figure 3 shows that in cursor space (left frames), group differences are evident at the $30 \mathrm{~mm}$ target amplitude, whereas in hand space (middle frames), differences emerged at the $230 \mathrm{~mm}$ target amplitude. These differences between the groups are important, because they show that participants in the transformation groups developed an internal model of the nonlinear transformation that was affected by intermediate amplitudes, rather than that each seen amplitude was independently translated into the associated movement amplitude. These observations were confirmed by two ANOVAs with the factors Group (notransformation, hand-same, cursor-same), and Amplitude $(30,230 \mathrm{~mm})$ carried out on the movement amplitudes in cursor space and hand space. The main effects of amplitude are not described because they simply reflect longer amplitudes at the $230 \mathrm{~mm}$ than the $30 \mathrm{~mm}$ amplitude.

The cursor space ANOVA showed a significant Group $\times$ Amplitude interaction, $F_{2,41}=4.1, P<0.05$, indicating that both transformation groups had longer amplitudes than the no-transformation group at the $30 \mathrm{~mm}$ amplitude (both $P<0.05)$, whereas there were no group differences at the $230 \mathrm{~mm}$ amplitude. In terms of deviation from the target amplitudes, there was a general overshoot of the amplitudes at the $30 \mathrm{~mm}$ amplitudes (ranging from $30 \mathrm{~mm}$ in the
A. Continuous Feedback

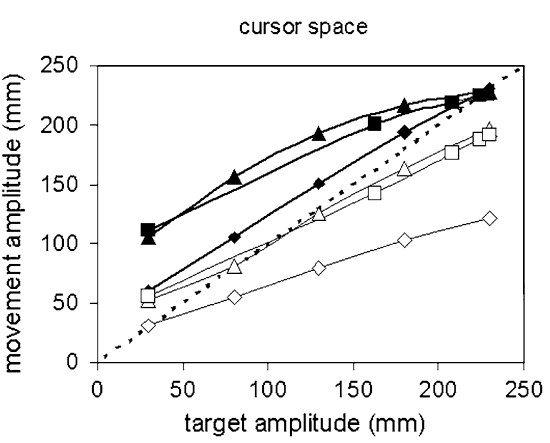

B. Terminal Feedback cursor space

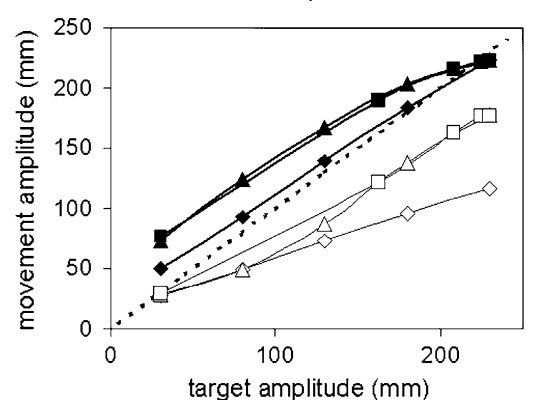

hand space

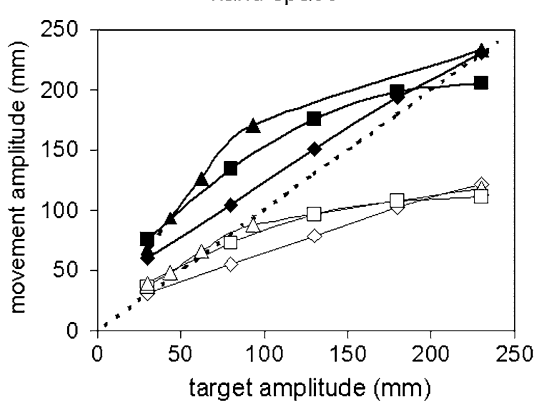

hand space

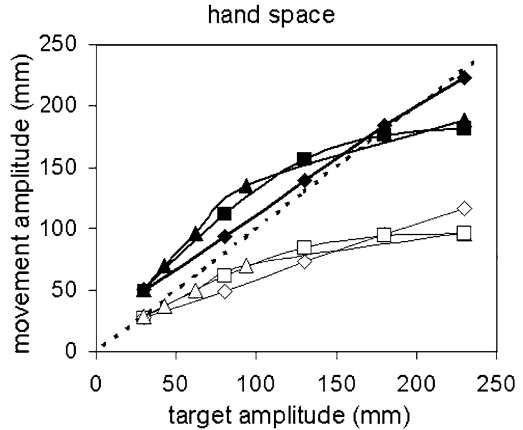

cursor to hand transformation
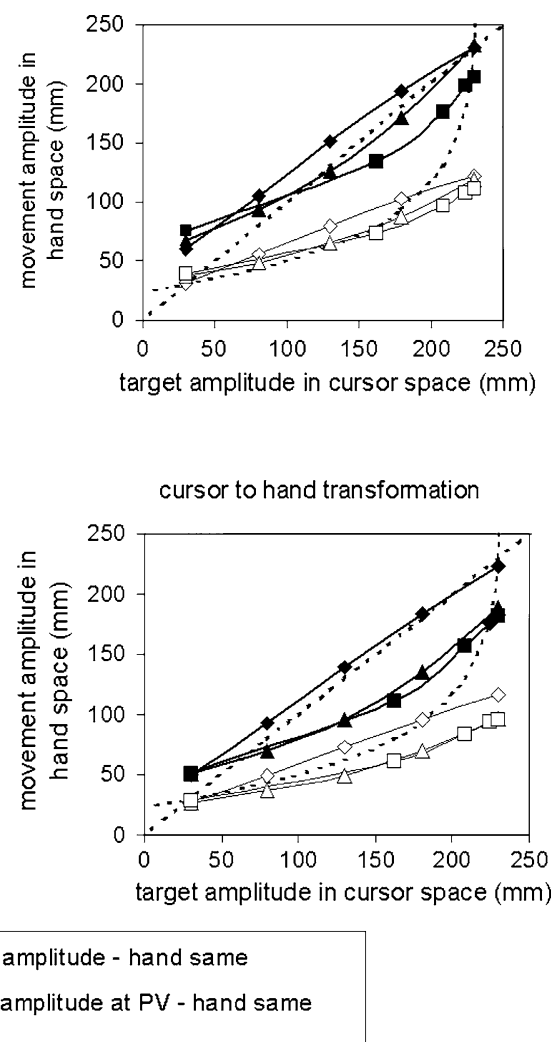

Fig. 3 Movement amplitude in cursor and in hand space as a function of transformation group and target amplitude in TRI bins $\geq 400 \mathrm{~ms}$. The lines with unfilled symbols indicate the movement amplitude at peak velocity. The right frames indicate deviations from the theoretical cursor to hand transformation (dashed lines) for the notransformation and the transformation groups. The theoretical transformation is the mathematically inverse function of the external transformation, which should be developed as an internal model 
no-transformation group to $81 \mathrm{~mm}$ in the hand-same group). At the $230 \mathrm{~mm}$ amplitude deviations were relatively minor ( -5 to $1 \mathrm{~mm})$.

The hand space ANOVA also showed a significant Amplitude $\times$ Group interaction, $F_{2,41}=3.8, P<0.05$, indicating that there were differences between the groups at the $230 \mathrm{~mm}$ amplitude, but not at the $30 \mathrm{~mm}$ amplitude. There was again an overshoot at the $30 \mathrm{~mm}$ amplitude (range 30-46 mm). Deviations at the $230 \mathrm{~mm}$ amplitude were relatively minor in the no-transformation group and the cursor-same group (1-3 $\mathrm{mm}$ ), however in the handsame group an undershoot of the target amplitudes was observable $(-24 \mathrm{~mm})$.

Level of operation of the internal model

The logic of determining the locus of operation of the internal model can be summarized as follows (for a more detailed description and simulations see Verwey and Heuer 2007). The time course of amplitude specification can be traced by forcing participants to start moving at different points in time before the process of amplitude specification has been completed. The resulting function shows how movement amplitude changes as a function of available preparation time (SOA, or actually TRI). Subsequently, this curve is expressed as changes between 0 (unchanged, mean movement amplitude at TRI bin $-105 \mathrm{~ms}$ ) and 1 (fully changed, mean amplitude at the three longest TRI bins).

An important assumption of the model and simulations (Verwey and Heuer 2007) is that the output of amplitude specification changes linearly with time (i.e., SOA/TRI) from the default to the target amplitude. If the internal model operates early (before or at amplitude specification) the actual movement amplitude changes linearly in hand space. When the movement amplitude is translated back to the accompanying amplitude in cursor space (expressed as relative change from 0 to 1 ), larger than default amplitudes (e.g., the $230 \mathrm{~mm}$ movement) initially increase faster with SOA than linear (producing a steeper curve), whereas smaller than default amplitudes (e.g., the $30 \mathrm{~mm}$ movement) initially increase more slowly than linear with SOA. Therefore, the difference in relative change of 30 and 230 $\mathrm{mm}$ amplitude movements is smaller in hand space than in cursor space. Conversely, when the internal model operates late (after amplitude specification), the relative change from default to target amplitude is linear in cursor space. This has the following effects in hand space: the initial change at short SOAs is especially large (yielding a steep curve) for smaller than default amplitudes (e.g., the $30 \mathrm{~mm}$ amplitude), and especially slow for larger than default amplitudes (e.g., the $230 \mathrm{~mm}$ amplitude). Thus, the difference in relative change of 30 and $230 \mathrm{~mm}$ amplitude movements is larger in hand space than in cursor space when the operation of the internal model follows amplitude specification. The model and simulations of Verwey and Heuer (2007) also takes into account that it usually takes longer to shorten a preplanned movement than to lengthen it, leading to an extra delay with amplitudes smaller than the default amplitude.

\section{Relative amplitudes}

In order to test these predictions, movement data were expressed as relative amplitudes from 0 to 1 , with 0 being the start point (i.e., mean movement amplitude at TRI bin $-105 \mathrm{~ms}$ ), and 1 the endpoint for that particular movement amplitude (mean amplitude of the three longest TRI bins). This yielded a gradual increase with TRI from 0 at TRI bin -105 to 1 at TRI bins $\geq 400$ ms. Expressing movements on a scale of 0-1 were done separately for each participant and each target amplitude both in hand and in cursor space. Again, we focused on the results of the 30 and $230 \mathrm{~mm}$ amplitudes, because they are comparable in cursor and hand space between all groups, and because they are less influenced by noise than intermediate amplitudes. Figure 4 shows relative amplitudes produced at these target amplitudes as a function of TRI (upper two rows). The difference between the 30 and $230 \mathrm{~mm}$ amplitudes is larger in cursor than in hand space, indicating that the internal model occurs before or at amplitude specification.

A Group (hand-same, cursor-same) $\times$ Bin (75, 165, 255 $\mathrm{ms}) \times$ Space (hand, cursor) ANOVA on the differences between relative amplitudes at the 30 and $230 \mathrm{~mm}$ target amplitude revealed a significant main effect of Space, $F_{1,29}=14.61, P<0.05$, indicating that differences were larger in cursor space (average difference: 0.31 ) than in hand space (0.11). There was also a significant main effect of Bin, $F_{2,58}=17.05, P<0.05$, reflecting that the differences between the 30 and $230 \mathrm{~mm}$ target amplitudes became successively smaller with longer TRI-bins. Thus, we obtained a steeper increase of the $230 \mathrm{~mm}$ amplitude than the $30 \mathrm{~mm}$ amplitude, and larger differences between the curves in cursor than in hand space. This indicates that the internal model operates before or at amplitude specification (Level 1 in Fig. 1).

\section{Amplitudes at peak velocity}

Another way of determining the locus of the internal model is by assessing whether it has any effect on the time course of trajectory generation, a parameter of which is the relative amplitude at which peak velocity occurs. Usually, this peak occurs about halfway the movement. If peak velocity occurs at approximately the same amplitude for all groups in hand space (implying differences in cursor space for the 

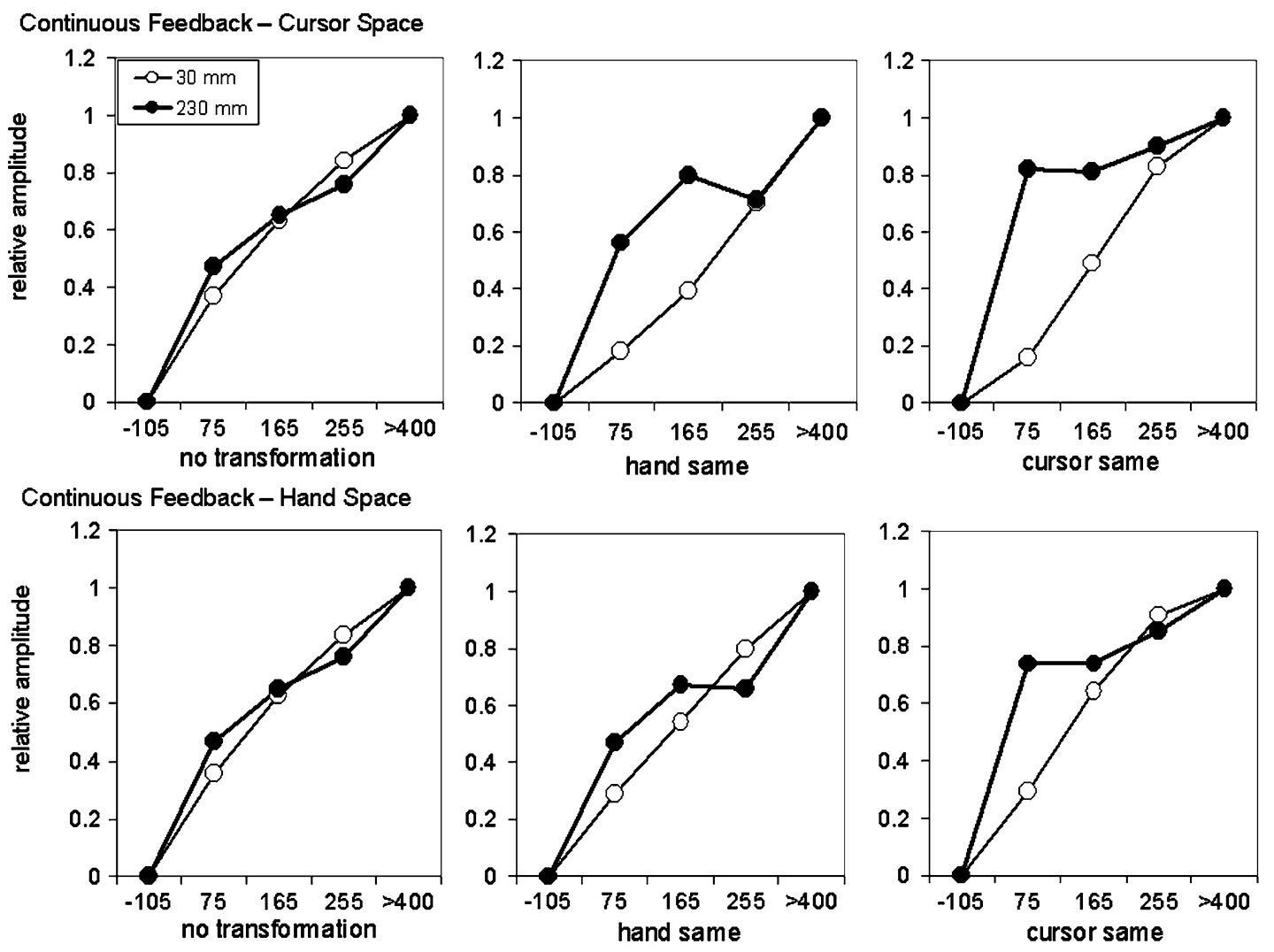

Terminal Feedback - Cursor Space
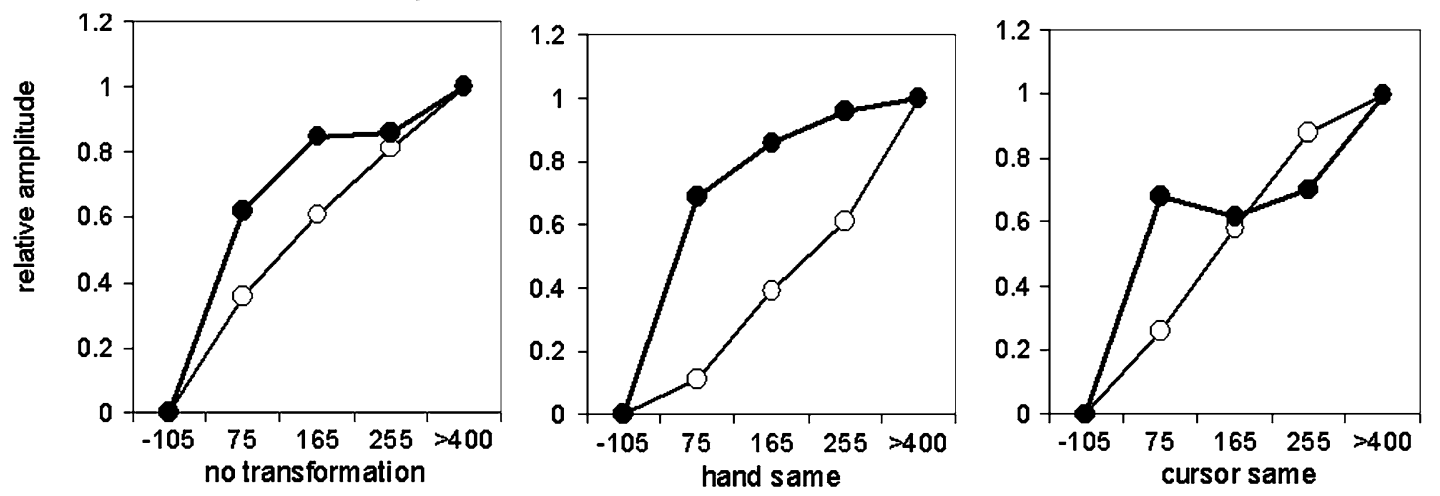

Terminal Feedback - Hand Space

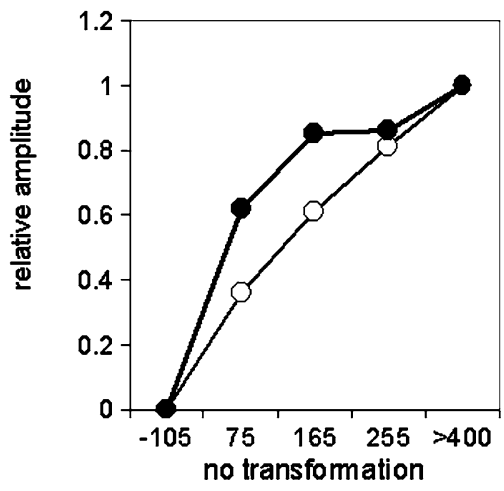

TRI bin
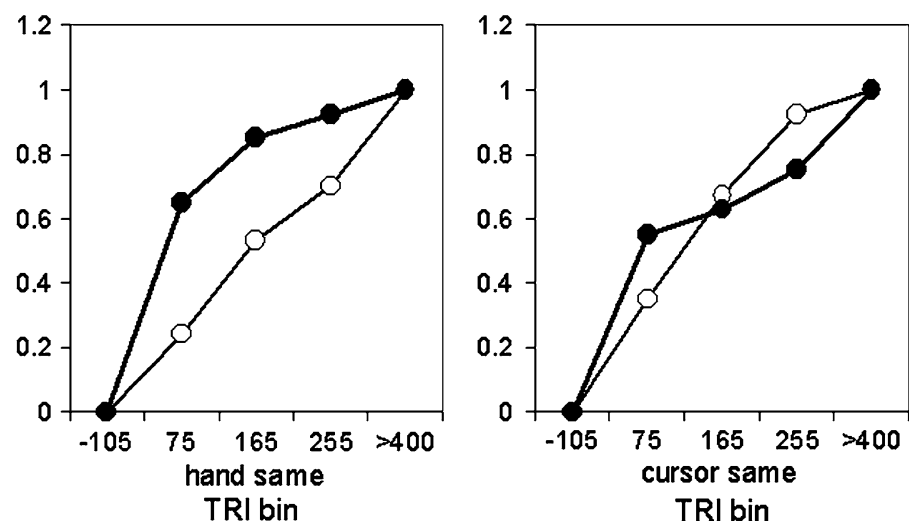

Fig. 4 Relative movement amplitudes for 30 and $230 \mathrm{~mm}$ target amplitudes as a function of TRI, space and group 
transformation groups), the internal model operates before trajectory generation. If peak velocity occurs at the same relative amplitude across groups in cursor space (implying differences in hand space for the transformation groups) the internal model operates during movement execution.

Figure 3 (open symbols, upper frames) shows that amplitudes at peak velocity are about half of the total amplitudes in hand space (middle frame), but not in cursor space (left frame). This supports that the internal model operates before or at trajectory generation. A Group (no-transformation, hand-same, cursor-same) $\times$ Amplitude (1:shortest amplitude,..., 5:longest amplitude) ANOVA on the percentage of amplitude at peak velocity in hand space confirmed this observation. Only a significant main effect of amplitude was observed, $F_{4,164}=7.3$, $P<0.05$, but no further significant differences. The percentage of amplitude at which peak velocity occurred was slightly higher for the shortest than for all other amplitudes (successive percentages were 53.9, 52.4, 52.4, $52.7,52.3 \%)$.

In contrast, the ANOVA in cursor space showed statistical significance for both main effects: Amplitude, $F_{4,164}=$ 134.4, $P<0.05$, and Group, $F_{2,41}=10.9, P<0.05$, as well as for the Group x Amplitude interaction, $F_{8,164}=40.7, P<$ 0.05 . In cursor space the percentage of the amplitude at which peak velocity occurred increased with amplitudes for both transformations groups (28.8-85.8\% in the cursorsame and $32.2-84.4 \%$ in the hand-same group from the shortest to the largest amplitude), whereas this was not the case for the no-transformation group $(53.9-52.5 \%$, see Fig. 3, left frames).

\section{Amplitude variability}

On the assumption that endpoint variability is caused primarily by motor noise during trajectory generation or later (Verwey and Heuer 2007) we examined whether movement variability across the three longest TRI bins was affected by the acquired transformation in hand space or in cursor space. The no-transformation group was compared to the hand-same group in hand space and to the cursorsame group in cursor space. The upper frames of Fig. 5 show that endpoint variability of the groups was similar in hand space, but not in cursor space. This provides additional evidence that the internal transformation preceded trajectory generation.

A Group (no-transformation vs. hand-same) $\times$ Amplitude $(30,80,130,180,230)$ ANOVA in hand space revealed a significant main effect of Group, $F_{1,27}=4.1, P<$ 0.05 , indicating that amplitude variability was slightly higher in the hand-same than in the no transformation group (21 vs. $14 \mathrm{~mm}$, respectively) and a significant main effect of Amplitude, $F_{4,108}=3.4, P<0.05$, indicating that amplitude variability decreased with larger amplitudes (22 at $30 \mathrm{~mm}$ amplitude vs. 16 at the $230 \mathrm{~mm}$ amplitude). In contrast, the ANOVA on amplitude variability in cursor space showed strong effects. A significant main effect of Amplitude, $F_{4,104}=35.6, P<0.05$, and a significant interaction of Group $\times$ Amplitude, $F_{4,104}=15, P<0.05$, indicated that whereas amplitude variability decreased considerably with increasing amplitude in the cursor-same group (from 37 at $30 \mathrm{~mm}$ amplitudes to 2 at $230 \mathrm{~mm}$ amplitude), this was less so the case for the no-transformation group (decrease from 19 to $13 \mathrm{~mm}$ ). The decreasing variability with larger amplitudes in cursor space is produced by the external transformation because differences in hand space have a larger effect on smaller than on larger cursor amplitudes. The slight reduction of endpoint variability with increasing amplitude in hand space may indicate that, as movements were larger (and took longer) feedback was used to improve endpoint accuracy.

\section{Awareness}

Figure 6 shows the amplitudes participants drew on paper to indicate the amplitudes they thought their hand had moved (in hand space) with each target amplitude (in cursor space). Further, the target amplitudes in hand and cursor space are shown. There was a substantial deviation in both transformation groups between the target amplitudes in hand space and the amplitudes participants reported to have moved. Participants in both transformation groups showed no awareness of the actual movement amplitudes and rather than movement amplitudes, they drew the amplitudes they had seen on the screen.

For the statistical analysis, differences were calculated between the subjective amplitudes and the target amplitudes in cursor and hand space for the two transformation groups. The differences were averaged across the three medium sized amplitudes (which differed in cursor and hand space). The average difference in cursor space was not significantly different from zero $(M=8, \mathrm{SD}=21 \mathrm{~mm})$, but the average difference in hand space was $(M=74, \mathrm{SD}=$ $\left.21 \mathrm{~mm}, t_{30}=19.9, P<0.05\right)$.

Additional results

\section{Movement times}

A Group (no-transformation, hand-same) $\times$ Amplitude (30, $80,130,180,230)$ ANOVA on average movement time across the three longest TRI bins showed only a main effect of amplitude, $F_{4,108}=115, P<0.05$, indicating that movement time increased from $241 \mathrm{~ms}$ with $30 \mathrm{~mm}$ to 422 ms with $230 \mathrm{~mm}$ movements. 
Fig. 5 Variability of movement amplitudes, averaged across the 3 longest TRI-bins, as a function of space and feedback condition. For better comparability only the notransformation and hand same group are depicted for hand space and the no-transformation and cursor same group are depicted in cursor space
A. Continuous Feedback

hand space
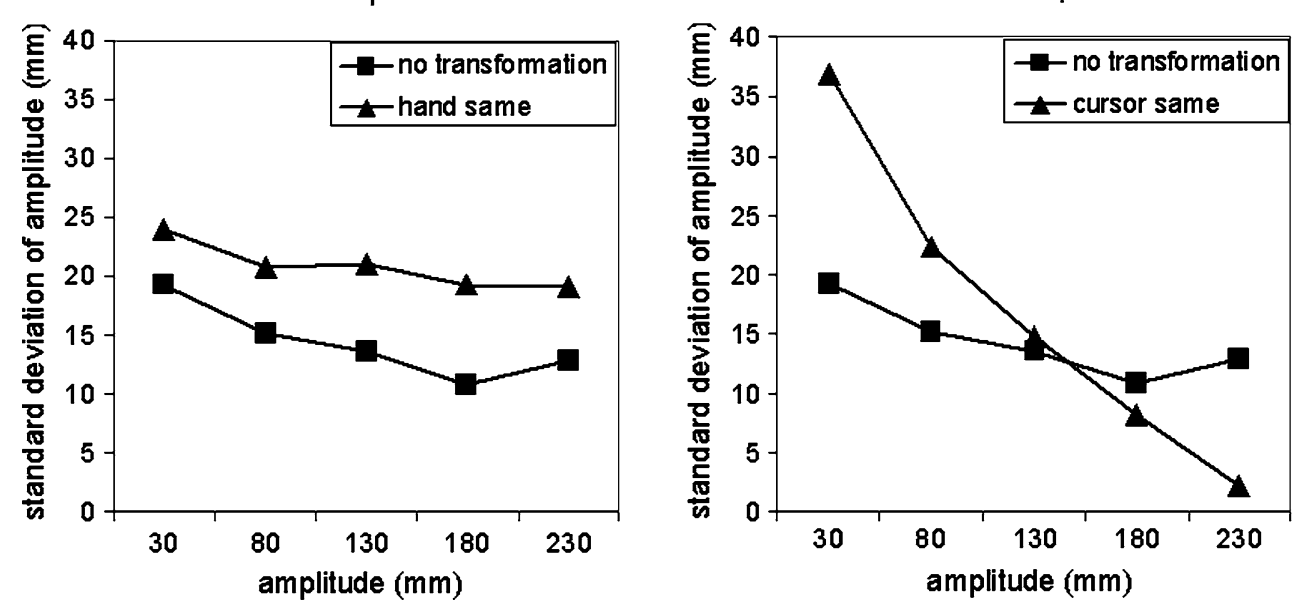

\section{B . Terminal Feedback}

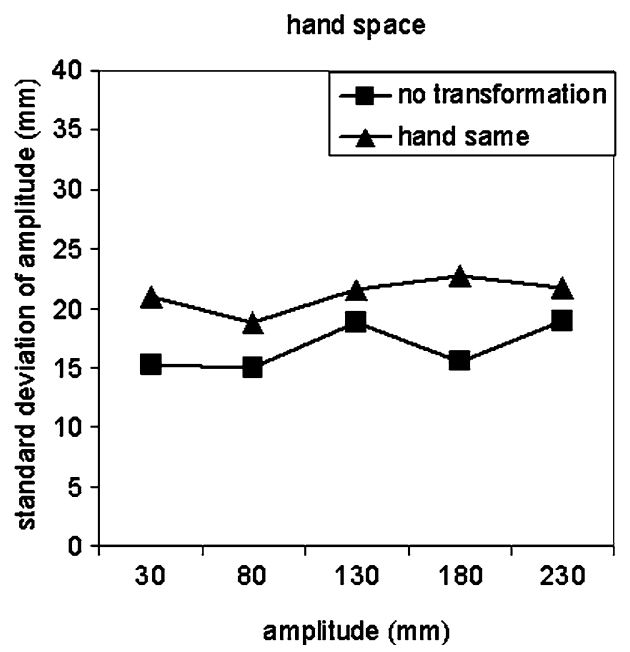

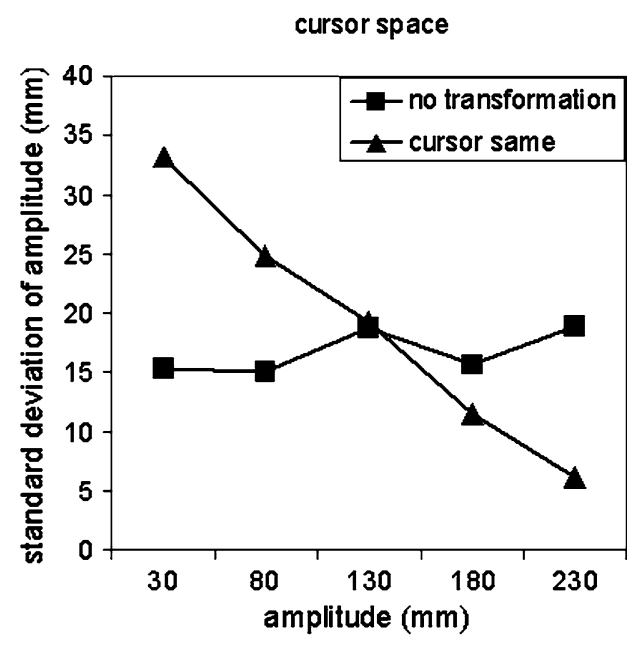

\section{Irregular trials}

For the $30 \mathrm{~mm}$ amplitude, the percentage of irregular trials was $4 \%$ and for the $230 \mathrm{~mm}$ amplitude the percentage of irregular trials was $26 \%$. Thus, the percentage of irregular trials was especially high with larger amplitudes. A Group (no-transformation, hand-same, cursor-same) $\times$ Amplitude $(30,230 \mathrm{~mm})$ ANOVA on percentage of irregular trials confirmed this by showing a main effect of Amplitude, $F_{1,41}=61.3, P<0.05$. These results are consistent with the notion that participants used visual feedback when there was sufficient time.

\section{Transfer to terminal feedback}

For the analysis of the terminal feedback data ANOVAs with the same factors as detailed above were carried out. Most striking was that the results showed the same overall pattern as those obtained with continuous feedback.
The internal model

The lower frames of Fig. 3 show the actual movement amplitudes in cursor and in hand space with terminal feedback. In cursor space, there were no systematic differences between the groups. In terms of deviation from the target amplitudes, there was a general overshoot of the amplitudes at the $30 \mathrm{~mm}$ amplitudes (ranging from $20 \mathrm{~mm}$ in the no-transformation group to $47 \mathrm{~mm}$ in the hand-same group). At the $230 \mathrm{~mm}$ amplitude, deviations were relatively minor (around -7 to $-8 \mathrm{~mm}$ ). In hand space there were a significant main effect of Group, $F_{2,41}=3.7$, $P<0.05$, and a significant interaction Amplitude $\mathrm{x}$ Group, $F_{2,41}=6.2, P<0.05$. There were no differences between the groups at the $30 \mathrm{~mm}$ amplitude. All groups overshot the target amplitude 20-21 mm. However, at the $230 \mathrm{~mm}$ amplitude the no-transformation group showed significantly longer amplitudes than both transformation groups (both $P<0.05$ ). Deviations from the target amplitude at the 


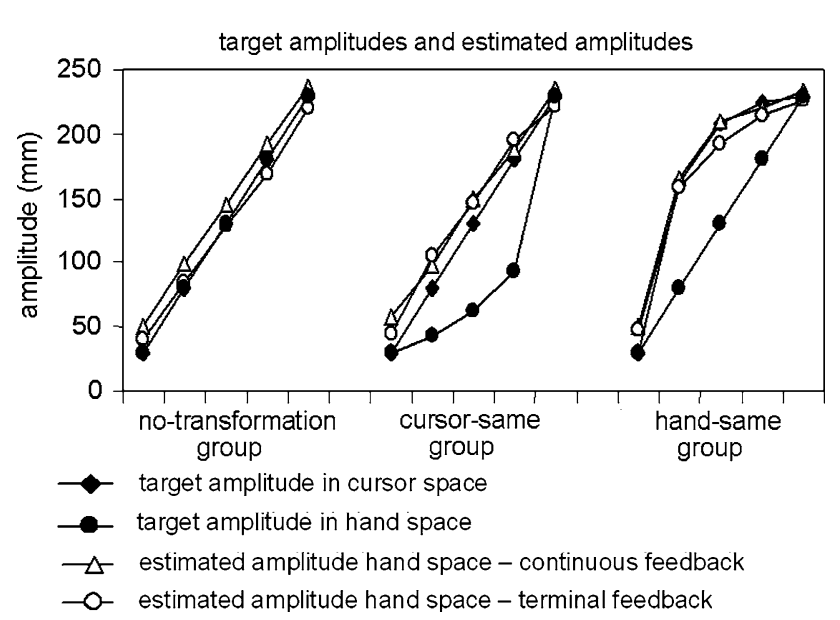

Fig. 6 Movement amplitudes as reported by the participants in the continuous and terminal feedback conditions in comparison to the formal target amplitudes in cursor and in hand space. Conditions are numbered from 1: shortest amplitude, to 5: longest amplitude

$230 \mathrm{~mm}$ amplitude were $-7 \mathrm{~mm}$ in the no-transformation group, $-41 \mathrm{~mm}$ in the cursor-same and $-48 \mathrm{~mm}$ in the hand-same group. Again, these results indicate that participants in the transformation groups developed an internal model of the nonlinear transformation that was affected but intermediate amplitudes, rather than that each seen amplitudes was independently translated into the associated movement amplitude.

\section{Level of operation of the internal model: relative amplitudes}

The data in the lower rows of Fig. 4 show the same pattern as the data for continuous feedback: larger differences between the $30 \mathrm{~mm}$ and the $230 \mathrm{~mm}$-curves in cursor space. The ANOVA showed a significant main effect of Space, $F_{1,29}=7.67, P<0.05$, confirming larger differences in cursor space $(0.27)$ than in hand space $(0.16)$. There were also main effects of Bin, $F_{2,58}=11.9, P<0.05$, reflecting smaller differences with longer TRI bins, and of Group, $F_{1,29}=12.77, P<0.05$, indicating that differences were more pronounced in the hand same group. These results suggest that the internal model operated before or at amplitude specification.

\section{Level of operation of the internal model: amplitudes at peak velocity}

Figure 3 (thin lines, lower frames) shows a similar picture for terminal feedback as for full feedback: the amplitudes at peak velocity are about halfway of the movement (middle frame) in hand space, but not in cursor space. The ANOVA on the percentage of amplitude at peak velocity in hand space showed again only a main effect of Amplitude,
$F_{4,164}=6.7, P<0.05$, but no further significant differences. The percentage of amplitude at which peak velocity occurred was slightly higher in the shorter than the longer amplitudes (successive percentages: 54.5, 53.5, 52.9, 52.7, $52.2 \%)$.

In contrast, the ANOVA in cursor space showed statistical significance for both main effects: Amplitude, $F_{4,164}=$ 192.6, $P<0.05$, and Group, $F_{2,41}=10.3, P<0.05$, as well as the interaction Group $\times$ Amplitude, $F_{8,164}=62.7, P<$ 0.05 . In cursor space, the percentage of the amplitude at which peak velocity occurred increased with larger amplitudes for both transformations groups (16-79.3\% in the cursor-same and $19.1-79.5 \%$ in the hand-same group from shortest to largest amplitude), whereas this was not the case for the no-transformation group (53.8-52.2\%). Thus, again the data show that amplitudes at peak velocity were about half the final amplitudes in hand space, but not in cursor space, indicating that the operation of the internal model precedes trajectory generation.

\section{Level of operation of the internal model: amplitude variability}

The lower frames of Fig. 5 show that again endpoint variability in the transformation groups was similar to that of the no-transformation group in hand space, but not in cursor space. The ANOVA on amplitude variability in hand space revealed no significant effects. The ANOVA in cursor space revealed a significant main effect of Amplitude, $F_{4,104}=10.4, P<0.05$, and a significant interaction of Group $\times$ Amplitude, $F_{4,104}=16.1, P<0.05$. Whereas amplitude variability decreased considerably with increasing amplitude in the cursor-same group (from 33 at $30 \mathrm{~mm}$ amplitudes to 6 at $230 \mathrm{~mm}$ amplitude), this was not the case for the no-transformation group (slight increase from 15 to $19 \mathrm{~mm}$ ). The decreasing variability with larger amplitudes in cursor space is again produced by the external transformation. In contrast to the continuous feedback conditions, no reduction of endpoint variability with increasing amplitude in hand space was found, supporting the interpretation above that feedback was used to improve endpoint accuracy. In short, the data indicate that the internal transformation preceded trajectory generation.

\section{Awareness}

The average difference between subjective and target amplitudes in cursor space was not significantly different from zero $(M=5, \mathrm{SD}=35 \mathrm{~mm})$, but the average difference in hand space was $\left(M=71, \mathrm{SD}=34 \mathrm{~mm}, t_{30}=11.7, P<0.05\right)$. Thus, again participants in the nonlinear groups showed no awareness of the actual movement amplitudes. Rather, they drew the amplitudes they had seen on the screen. 


\section{Additional results}

The ANOVA on movement times revealed only a significant main effect of amplitude, $F_{4,108}=136.4, P<0.05$, indicating that movement time increased from $215 \mathrm{~ms}$ with $30 \mathrm{~mm}$ to $407 \mathrm{~ms}$ with $230 \mathrm{~mm}$ movements.

The percentages of irregular trials were $1.5 \%$ at the 30 $\mathrm{mm}$ amplitude and $8 \%$ at the $230 \mathrm{~mm}$ amplitude. This increase was confirmed by a significant main effect of Amplitude, $F_{1,41}=38.7, P<0.05$. The percentage of irregular trials at the $230 \mathrm{~mm}$ amplitude was, however, much lower than with continuous feedback, $t(43)=7.1, P<0.05$.

\section{Discussion}

In the present study, participants performed a manual aiming task either when a nonlinear relationship existed between the hand and cursor movement or when no transformation existed. They practiced the task with continuous feedback first, and subsequently executed it with terminal feedback. After both conditions, an awareness test was conducted. Results showed that (a) participants in the experimental conditions developed a nonlinear internal model, (b) the acquired internal model operated at or before amplitude specification under both feedback conditions, (c) continuous feedback did affect movement control, and (d) participants did not show awareness of the actual transformation.

The acquired internal model was similar for both feedback conditions and showed imperfections. The imperfections at the 30 and $230 \mathrm{~mm}$ amplitudes are in line with the idea that the internal model consists of a continuous rule (cf. Bedford 1989; Hay 1974; Verwey and Heuer 2007) rather than individual stimulus-response mappings for all amplitudes (as if it were a lookup table). The analyses of relative amplitudes across TRI bins, percentage of amplitude at peak velocity and amplitude variability all indicate that under both feedback conditions, the level of operation of the internal model was at or before amplitude specification. First, relative amplitude differences between larger and smaller amplitudes were larger in cursor space than in hand space resulting in a more bulky shape in cursor space. Second, in the transformation groups the percentage of amplitude at which peak velocity occurred was essentially the same in hand space for amplitudes of different lengths, but not in cursor space, indicating that trajectory generation was not affected by the internal model. Third, amplitude variability was similar across groups in hand space, but was affected by the internal model in cursor space in the transformation groups. These findings provide converging evidence that the internal model operates at or before amplitude specification in both feedback conditions.
It was somewhat surprising that with continuous feedback the internal model operated at or before amplitude specification, even though systematic differences between continuous and terminal feedback conditions (e.g., amplitude variability) indicate that participants did use the feedback to a certain extent (despite time pressure and the requirement to move smoothly). It was less surprising that the internal model operated at or before amplitude specification in the terminal feedback condition as that has been found by Verwey and Heuer (2007) too. We originally included this condition to see whether the locus of the internal model would change if continuous feedback training would yield another locus than in Verwey and Heuer (2007).

Importantly, participants in the transformation groups were not aware of the actual amplitudes they had moved under continuous feedback, even though they had been told in advance that there would not necessarily be a 1:1 relationship between hand and cursor space. Indeed, some participants spontaneously reported that "something weird" was going on, however, they did not describe the nonlinear relationship. One explanation may be that participants were prevented from noticing the exact relationship between ongoing movement of the hand and the cursor, because hand movements were rapid and not visible. We cannot exclude that with slower movements awareness would have developed. However, proprioception in general does not seem to be very accurate in predicting hand position (Ghilardi et al. 1995), and proprioceptive feedback might even be reduced during the adaptation to visuomotor transformations (Jones et al. 2001; Bernier et al. 2005). In fact, it seems rather common that conscious awareness of transformations is limited (Knoblich and Kircher 2004).

In conclusion, when executing rapid movements with continuous feedback, the level of operation of an internal model adjusting for nonlinear transformations operates at or before amplitude specification. Taken together with previous results by Verwey and Heuer (2007) and the results from our terminal feedback condition, we may conclude that with rapid movements the type of feedback does not matter for the locus of the internal model adjusting for nonlinear transformations: it is before or at amplitude specification.

Open Access This article is distributed under the terms of the Creative Commons Attribution Noncommercial License which permits any noncommercial use, distribution, and reproduction in any medium, provided the original author(s) and source are credited.

\section{References}

Bedford FL (1989) Constraints on learning new mappings between perceptual dimensions. J Exp Psychol Hum Percept Perform $15: 232-248$ 
Bedford FL (1994) Of computer mice and men. Cah Psychol Cogn 13:405-426

Bernier PM, Chua R, Franks IM (2005) Is proprioception calibrated during visually guided movements? Exp Brain Res 167:292-296

Bock O, Burghoff M (1997) Visuo-motor adaptation: evidence for a distributed amplitude control system. Behav Brain Res 89:267-273

Cohen MM (1967) Continuous versus terminal visual feedback in prism aftereffects. Percept Mot Skills 24:1295-1302

Flanagan JR, Rao AK (1995) Trajectory adaptation to a nonlinear visuomotor transformation: evidence of motion planning in visually perceived space. J Neurophysiol 74:2174-2178

Ghahramani Z, Wolpert DM, Jordan MI (1996) Generalization to local remappings of the visuomotor coordinate transformation. J Neurosci 16:7085-7096

Ghilardi MF, Gordon J, Ghez C (1995) Learning a visuomotor transformation in a local area of work space produces directional biases in other areas. J Neurophysiol 73:2535-2539

Hay JC (1974) Motor-transformation learning. Perception 3:487-496

Hening W, Favilla M, Ghez C (1988) Trajectory control in targeted force impulses V. Gradual specification of response amplitude. Exp Brain Res 71:116-128

Heuer H (2003) Motor control. In: Healy AF, Proctor RW (eds) Handbook of psychology, vol 4. Experimental psychology. Wiley, New York, pp 317-354

Heuer H, Hegele M (2007) Learning new visuo-motor gains at early and late working age. Ergonomics 50:979-1003

Imamizu H, Miyauchi S, Tamada T, Sasaki Y, Takino R, Putz B, Yoshioka T, Kawato M (2000) Human cerebellar activity reflecting an acquired internal model of a new tool. Nature 403:192-195

Jones KE, Wessberg J, Valbo A (2001) Proprioceptive feedback is reduced during adaptation to a visuomotor transformation: preliminary findings. Neuroreport 12:4029-4033

Kagerer FA, Contreras-Vidal JL, Stelmach GE (1997) Adaptation to gradual as compared with sudden visuo-motor distortions. Exp Brain Res 115:557-561

Knoblich G, Kircher TTJ (2004) Deceiving oneself about control: conscious detection of changes in visuomotor coupling. J Exp Psychol Hum Percept Perform 30:657-666

Massen C, Prinz W (2007) Programming tool-use actions. J Exp Psychol Hum Percept Perform 33:692-704

Mottet D, Bardy BG, Athenes S (1994) A note on data smoothing for movement analysis: the relevance of a nonlinear method. J Motor Behav 26:51-55

Rieger M, Knoblich G, Prinz W (2005) Compensation of and adaptation to sudden changes in the environment. Exp Brain Res 163:487-502

Seidler RD, Bloomber JJ, Stelmach GE (2001) Patterns of transfer of adaptation among body segments. Behav Brain Res 122:145-157

Verwey WB, Heuer H (2007) Nonlinear visuomotor transformations: locus and modularity. Q J Exp Psychol 60:1629-1659

Willingham DB (1998) A neuropsychological theory of motor skill learning. Psychol Rev 105:558-584

Wolpert DM, Ghahramani Z, Jordan MI (1995) Are arm trajectories planned in kinematic or dynamic coordinates? An adaptation study. Exp Brain Res 103:460-470 